\title{
Pulmonary immune response to Streptococcus pneumoniae
}

\author{
Jimstan Periselneris \\ Ricardo J. José \\ Jeremy S. Brown
}

University College London, London, UK

\author{
Address for correspondence: \\ Jimstan Periselneris, MBChB \\ University College London \\ London, UK \\ E-mail: j.periselneris@ucl.ac.uk
}

\begin{abstract}
Summary
Streptococcus pneumoniae is a common cause of respiratory infection associated with significant morbidity and mortality. The immune response to S. pneumoniae is complex, multi-layered and incompletely understood. The lung has non-specific defence mechanisms that include cellular and secreted components. The initial response to bacteria that adhere to or breach the epithelium is mediated by the innate immune system and recognition of non-specific motifs common to many pathogens. This stimulates the inflammatory response and subsequently the development of the more specific adaptive immune system. Better understanding of the immune response to $S$. pneumoniae will underpin future progress in both the prevention of infection and adjuvants to existing treatments.
\end{abstract}

KEY WORDS: Streptococcus pneumoniae; immunology.

\section{Introduction}

Streptococcus pneumoniae is the commonest cause of pneumonia and is thought to be responsible for over a million deaths per year, particularly in children and the elderly (1). Despite ef-

Despite effective antibiotics, S. pneumoniae accounts for $33 \%$ of vaccine preventable deaths due to pneumonia in children worldwide. fective antibiotics $S$. pneumoniae accounts for $33 \%$ of vaccine preventable deaths due to pneumonia in children worldwide (2). Community acquired pneumonia (CAP) is also associated with substantial morbidity and high economic burden, costing 10.1 billion euros per year in Europe (3). Host factors that are known to increase the risk of pneumococcal infection include immune deficiency (e.g. HIV infection, immunoglobulin deficiencies, complement deficiencies, myeloma, post organ transplant), splenic dysfunction (e.g. asplenia, sickle cell disease), and chronic organ dysfunction (e.g. liver cirrhosis and chronic lung disease) (4). The extremes of age, smoking, alcohol abuse, and influenza also increase the risk of contracting disease. Given the importance of this bacterium in lung disease, understanding the pulmonary host immune response is essential for the design of more effective therapeutic interventions.

\section{S. pneumoniae biology}

S. pneumoniae is a gram positive lancet shaped bacterium that usually grows as diplococci. It is a nasopharyngeal commensal, a site where it does not cause significant pathology. Rates of colonisation are particularly high amongst nursery age children, but reduce to approximately $10 \%$ in healthy adults (5). S. pneumoniae reaches the lower respiratory tract after microaspiration and are much more likely to cause infection when pulmonary host defence mechanisms are disrupted. Invasion
S. pneumoniae needs to adhere to respiratory epithelium, so infection is mediated by the interactions of bacterial and host cell surface structures. through epithelial layers either locally from the nasopharynx or from the lung during pneumonia can lead to septicaemia.

All virulent strains of $S$. pneumoniae express a polysaccharide capsule that surrounds a cell wall consisting of peptidoglycan and teichoic acid. The capsule's primary function is to inhibit opsonophagocytosis by the host immune system (6). There are over 90 different serotypes, classified by the composition of the oligosaccharide makeup of the capsule. Duration of colonisation, invasiveness, and case-fatality rates vary with capsular serotype, for example serotypes 3 , $6 \mathrm{~A}, 6 \mathrm{~B}, 9 \mathrm{~N}, 19 \mathrm{~F}, 23 \mathrm{~F}, 31$ are overrepresented in fatal cases of infection $(7,8)$. Other important $S$. pneumoniae virulence factors are a range of secreted and cell wall-associated proteins. These include the toxin pneumolysin that has pleiotropic effects on the host immune response including cell lysis, complement activation, inhibition of neutrophil function, and increased inflammation, as well as cell wall proteins that inhibit opsonophagocytosis. In addition, to achieve 
successful infection, S. pneumoniae needs to adhere to respiratory epithelium, which is mediated by the interactions of bacterial and host cell surface structures. These include hydrophobic and electrostatic surface characteristics contributing to physiochemical interactions, and S. pneumoniae's ability to bind to N-acetylgalactosamine on resting epithelium (9), the ability of the cell wall protein PspC (pneumococcal surface protein $\mathrm{C}$ ) to bind to the laminin receptor expressed on a variety of host cell types (10), and cell wall phosphocholine which binds to the platelet activating factor receptor (11). Bacterial/host cell interactions also stimulate active bacterial translocation across epithelial and endothelial layers to reach the blood or central nervous systems (12).

\section{Host defence mechanisms}

The pulmonary host defence mechanisms to $S$. pneuDamage to physical
defence mechanisms
through viral infec-
tion of the epithelium
or inhibition of mu-
cociliary function by
cigarette smoke in-
crease the ability of
invading bacteria to
establish infection
within the lungs. moniae are multi-layered and involve many complex interactions between separate components of the immune system. Initial physical pulmonary defences include the mucociliary escalator and the integrity of the pulmonary epithelium. Mucus entraps pathogens, inhibiting adherence of the bacteria to the underlying epithelium, and the action of cilia moves the entrapped pathogens out of the lung. An intact epithelium prevents bacterial adhesion to extracellular matrix and penetration into the lung interstitium and eventually the blood. Damage to these physical defences through viral infection of the epithelium (13) or inhibition of mucociliary function by cigarette smoke (14) increase the ability of invading bacteria to establish infection within the lungs. $S$. pneumoniae is a classic extracellular pathogen that replicates and persists mainly outside of host cells; hence the killing of $S$. pneumoniae that manage to evade physical defences is largely mediated by opsonophagocytosis and secreted proteins and peptides in epithelial lining fluid. To assist bacterial clearance, $S$. pneumoniae reaching the lungs stimulate an inflammatory response that recruits and activates immune cells to the lungs, including phagocytes. Additionally, an acute phase response is initiated allowing soluble serum mediators such as complement to enter the lung compartment following an increase in vascular permeability. Previous colonisation means that as well as the innate immune response all humans have some degree of adaptive immunity that can recognise $S$. pneumoniae and potentially assist bacterial clearance from the lungs. The most important aspects of the innate and adaptive immune response will be discussed separately below, although in reality they act in concert as an integrated system for prevention and control of $S$. pneumoniae persistence within the lung.

\section{Innate immune response}

\section{Epithelial cells and surfactant}

Type II pneumocytes secrete antibacterial products into alveolar lining fluid which assists with bacterial killing. These include: (a) lactoferrin, which sequesters free iron required for bacterial growth and oxidises bacterial cell membranes; (b) lysozyme, which breaks down peptidoglycan in bacterial cell walls; and (c) a wide range of antimicrobial peptides (e.g. defensins and cathelicidin) that form pores in bacterial cell surfaces thereby lysing invading pathogens. The efficacy of these soluble components of mucosal defences against $S$. pneumoniae has in general been poorly defined. $S$. pneumoniae is rela-
Alveolar macrophages are likely to be the cell type that primarily recognize $\mathrm{S}$. pneumonia, and then stimulate the alveolar epithelium to amplify the inflammatory response. tively resistant to lactoferrin as it produces a cell wall protein, pneumococcal surface protein A (PspA), that binds to lactoferrin and blocks its activity (15). Similarly, while lysozyme can restrict the growth of $S$. pneumoniae, modifications to its cell wall structure render it relatively resistant to degradation $(16,17)$. However, the antimicrobial peptide $\beta$-defensin- 2 does cause $S$. pneumoniae cell lysis and can act synergistically with lysozyme in controlling bacterial numbers (17). As well as secreting soluble antibacterial factors, epithelial cells express pattern recognition receptors (PRR) that recognise conserved molecular patterns found on the surface of microbial pathogens, including S. pneumoniae. Activation of epithelial PRRs can contribute towards the inflammatory response to $S$. pneumoniae (18). However, epithelial pro-inflammatory responses can be indirectly increased by macrophages (19). This suggests that alveolar macrophages are likely to be the cell type that primarily recognise $S$. pneumoniae, and then stimulate the alveolar epithelium by paracrine or juxtacrine mechanisms to amplify the inflammatory response, and attract required additional cells such as neutrophils to the site of infection.

Surfactant protein A \& D (SP-A \& D respectively) are constitutively synthesized and secreted into alveolar lining fluid by type II pneumocytes and non-ciliated bronchial epithelial cells. SP-A \& D bind to exposed mannose and glucose residues on the surface of bacteria, and this leads to agglutination of pathogens, inhibition of microbial growth, and increase recruitment of phagocytes. SP-A has also been shown to be an opsonin that increases alveolar macrophage phagocytosis of S. pneumoniae (20).

\section{Alveolar macrophages}

Macrophages are tissue resident phagocytes that play an important role in orchestrating innate immune responses to pathogens. Alveolar macrophages make up most of the resident leukocyte population in the alveolar space. The vital role for alveolar macropha- 
ges for preventing $S$. pneumoniae pneumonia is suggested by the increased incidence of pneumonia in humans with impaired macrophage function due to exposure to cigarette smoke, viral infection, corticosteroids, welding fumes, or alcohol (21-25). Alveolar macrophages are able to phagocytose foreign material, coordinate an inflammatory cytokine response to infection and act as antigen presenting cells. They kill pathogens by a variety of mechanisms, including the generation of oxidative species and serine proteases. Despite their important role as first-line of cells in pulmonary host defence, alveolar macrophages exhibit less phagocytic activity and respiratory burst than other types of macrophages, and are less likely to stimulate $\mathrm{T}$ cells; these phenotypes help to promote tolerance to innocuous antigens that reach the respiratory tract. In addition alveolar macrophages, by secreting TGF $\beta$ and prostaglandins may drive differentiation of $T$ cells into a regulatory phenotype that suppress inflammation (26). This reflects the important role alveolar macrophages have for maintaining homeostasis within the alveolar compartment, preventing continuous or uncontrolled inflammatory responses within the lung to the almost constant exposure to external antigens that occurs, in order to avoid the deleterious effects of inflammation on the delicate lung tissue.

Despite their relative anti-inflammatory resting state, alveolar macrophages express an array of PRRs including indirect phagocytic receptors such as Fc receptors for immunoglobulins and complement receptors for the activated complement components C3b and $\mathrm{iC} 3 \mathrm{~b}$ bound to the surface of invading pathogens, direct phagocytic receptors including lectins (dectin-1 and DC-SIGN), and scavenger receptors (SR-A and MARCO), and direct non- phagocytic receptors such as toll-like receptors (TLR). Through phagocytic receptors alveolar macrophages can ingest and kill invading bacteria, and evidence from murine models suggest they can rapidly clear low numbers of $S$. pneumoniae from the lungs (27). Hence, alveolar macrophages can deal with low levels of infection by phagocytosis. However, when large numbers of $S$. pneumoniae reach the lung they overwhelm alveolar macrophage mediated clearance. In response, alveolar macrophages initiate an inflammatory response through the release of pro-inflammatory cytokines including TNF, IL6, and IL-1 $\beta$ and chemokines $(19,28)$, thereby recruiting additional mechanisms of microbial clearance, such as neutrophils, to the lung. Conversely, anti-inflammatory cytokines released by alveolar macrophages are necessary for resolution of inflammation (29). Furthermore, following neutrophil clearance of infection, macrophages also remove apoptotic neutrophils thereby assisting with resolution of inflammation $(29,30)$; failure of alveolar macrophages to clear neutrophils results in neutrophil necrosis, the release of reactive oxygen species and proteases, and subsequent tissue injury and inflammation (31). The ability of alveolar macrophages to limit inflammation for the majority of the time, but elicit an inflammatory response to a significant invasion by pathogens may be mediated by their close relationship with alveolar ep-

ithelium. This allows negative regulators present on the alveolar epithelium (e.g. the surface proteins CD200 on CD200R) to inhibit alveolar macrophage-mediated inflammation, but the inhibitory effect is lost after epithelial damage.

\section{Neutrophils}

Neutrophils or polymorphonuclear leukocytes are the most abundant leukocyte population and are usually the initial cells recruited to sites of injury and infection. Although there are very few neutrophils present on the epithelial surface or interstitium of normal lungs, the pulmonary capillary bed contains a significant reservoir of intravascular neutrophils that are rapidly recruited to the alveolar space during poorly controlled bacterial infection. Recruited neutrophils play a crucial role in host defence to bacterial pathogens, as demonstrated by an increased incidence of $S$. pneumoniae pneumonia when neutrophil function is impaired (e.g. Chediak-Higashi syndrome where there is failure of phagolysosome formation and reduced lysosomal degranulation) or neutrophil numbers are reduced (32, 33). On the other hand, neutrophilic inflammation is also implicated in tissue damage that can result in lung injury and the develop-

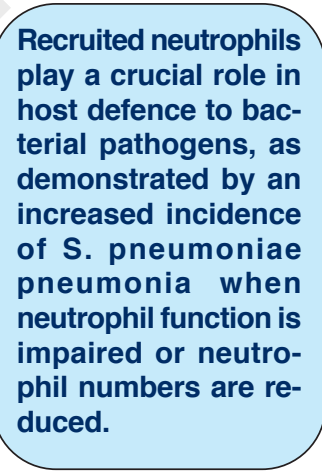
Recruited neutrophils play a crucial role in host defence to bacterial pathogens, as demonstrated by an increased incidence of S. pneumoniae pneumonia when neutrophil function is impaired or neutro duced. ment of the acute respiratory distress syndrome. Additionally, they have been implicated in the development of bacteraemia, with some murine studies suggesting that neutropenia is associated with reduced bacteraemia and prolonged survival $(19,34)$.

Neutrophils primarily engage with pathogens via phagocytosis followed by the formation of phagolysosomes and intracellular killing. Neutrophil phagocytosis of $S$. pneumoniae is highly dependent on recognition of the bacteria by proteins of the complement system or by specific antibody $(6,35)$. Neutrophils also produce neutrophil extracellular traps (NETs) by extruding their chromatin and DNA to form extracellular filaments that entrap pathogens and expose them to antimicrobial compounds. However, $S$. pneumoniae is relatively resistant to NETs by virtue of its capsule and expression of surface endonucleases that degrade DNA (36). S. pneumoniae is also resistant to the main mechanism of bacterial killing by neutrophils, the NADPH oxidase-dependent oxidative burst that generates reactive oxygen. As a result, humans with chronic granulomatous disease, who fail to produce ROS, don't have excessive rates of S. pneumoniae infection. Instead, S. pneumoniae killing by neutrophils is mediated by azurophilic granules that contain the serine proteases cathepsin $\mathrm{G}$ and neutrophil elastase which degrade bacterial proteins $(37,38)$, defensins (17), and bactericidal permeability-increasing protein (39). 


\section{J. Periselneris et al.}

Table 1 - PRRs and their potential S. pneumoniae ligand/activating molecule.

\begin{tabular}{|c|c|c|c|}
\hline PRR & $\begin{array}{l}\text { Where PRR is } \\
\text { expressed }\end{array}$ & S. pneumoniae ligand & $\begin{array}{l}\text { Consequences of } \\
\text { activation }\end{array}$ \\
\hline$\overline{T L R} 2$ & $\begin{array}{l}\text { Leucocyte and epithelial } \\
\text { cell surface }\end{array}$ & $\begin{array}{l}\text { Lipoproteins } \\
\text { Cell wall constituents? }\end{array}$ & $\begin{array}{l}\text { Proinflammatory } \\
\text { cytokine transcription }\end{array}$ \\
\hline TLR 4 & $\begin{array}{l}\text { Leucocyte and epithelial } \\
\text { cell surface }\end{array}$ & Pneumolysin? & $\begin{array}{l}\text { Proinflammatory } \\
\text { cytokine transcription }\end{array}$ \\
\hline TLR 9 & Leucocyte endosomes & $\begin{array}{l}\text { Unmethylated CpG } \\
\text { DNA fragments }\end{array}$ & $\begin{array}{l}\text { Proinflammatory } \\
\text { cytokine transcription } \\
\text { Type } 1 \text { IFN transcription }\end{array}$ \\
\hline NOD 2 & Leucocyte cytoplasm & $\begin{array}{l}\text { Cell wall } \\
\text { muramyldipeptide }\end{array}$ & $\begin{array}{l}\text { Proinflammatory } \\
\text { cytokine transcription }\end{array}$ \\
\hline NLRP 3 & Cytoplasm & Pneumolysin? indirect & $\begin{array}{l}\text { Activation of pro IL1 } \beta \\
\text { and IL18 }\end{array}$ \\
\hline DC-SIGN / SIGN-R1 & $\begin{array}{l}\text { Macrophages and } \\
\text { dendritic cells (marginal } \\
\text { zone of spleen) }\end{array}$ & $\begin{array}{l}\text { Capsular } \\
\text { polysaccharide? }\end{array}$ & $\begin{array}{l}\text { Increased phagocytosis } \\
\text { Complement activation? } \\
\text { Improved natural IgM } \\
\text { production? }\end{array}$ \\
\hline Unknown & Cytoplasm & Bacterial DNA & $\begin{array}{l}\text { Activation of STING to } \\
\text { induce Type } 1 \text { IFN } \\
\text { transcription }\end{array}$ \\
\hline MARCO & Macrophages & Unknown & $\begin{array}{l}\text { Increased phagocytosis } \\
\text { Amplification of other }\end{array}$ \\
\hline Scavenger receptors & Macrophages & Unknown & Increased phagocytosis \\
\hline $\begin{array}{l}\text { SP-A \& SP-D surfactant } \\
\text { proteins }\end{array}$ & $\begin{array}{l}\text { Release into epithelial } \\
\text { lining fluid by Type II } \\
\text { pneumocytes and Clara } \\
\text { cells }\end{array}$ & $\begin{array}{l}\text { Surface mannose \& } \\
\text { glucose residues }\end{array}$ & Increased phagocytosis \\
\hline
\end{tabular}

\section{Pattern recognition receptors}

The host immune system first recognises S. pneumoniae via PRR expressed in epithelial cells and macrophages within the respiratory tract (Table 1 ). These receptors recognise pathogen associated molecular patterns (PAMP) such as components of the pneumococcal cell membrane (lipoproteins) (40), cell wall (peptidoglycan and lipoteichoic acid), and also recognise damage associated molecular patterns (DAMPs e.g. the HGMB1 and S100 host proteins) released by endogenous cells as a result of tissue damage (Figure 1). PRRs are often situated on cell surface membranes, but are also present on endosomes and as such are in position to recognise PAMPs released into phagolysosomes by bacterial degradation. The most important PRRs for alveolar macrophage interactions with $S$. pneumoniae are described in more detail below.

\section{Toll-like-receptors}

The most important non-phagocytic PRRs for $S$. pneumoniae belong to the Toll-like-receptors (TLR) family of single, membrane-spanning, non-catalytic proteins. TLR 1, 2, 4, 5, and 6 are situated on the cell surface membrane and the rest reside on endosomes. When they recognise PAMPs they activate cell signalling pathways by a number of adaptor molecules (MyD88, TRAP, TRIF, and MAL) that culminate in altered gene transcription. Children with interleukin-1 receptor-associated kinase 4 (IRAK4) and similar deficiencies that affect TLR intracellular signalling pathways have impaired macrophage pro-inflammatory responses and high incidences of often lethal $S$. pneumoniae infections (41).

TLR 2 forms heterodimers with TLR6 to recognise diacylated lipoproteins, proteins that are covalently anchored to the bacterial cell membrane, including $S$. pneumoniae $(40,42,43)$. The importance of TLR2 in induction of the inflammatory response to $S$. pneumoniae has been demonstrated in a mouse model of infection with TLR2-/- mice having decreased cytokine (e.g. IL1 $\beta$, IL6, and KC, the mouse homologue of CXCL8) responses to $S$. pneumoniae. Recent data suggests that lipoproteins are important TLR2 ligands that are required for IRAK4 mediated protective inflamma- 


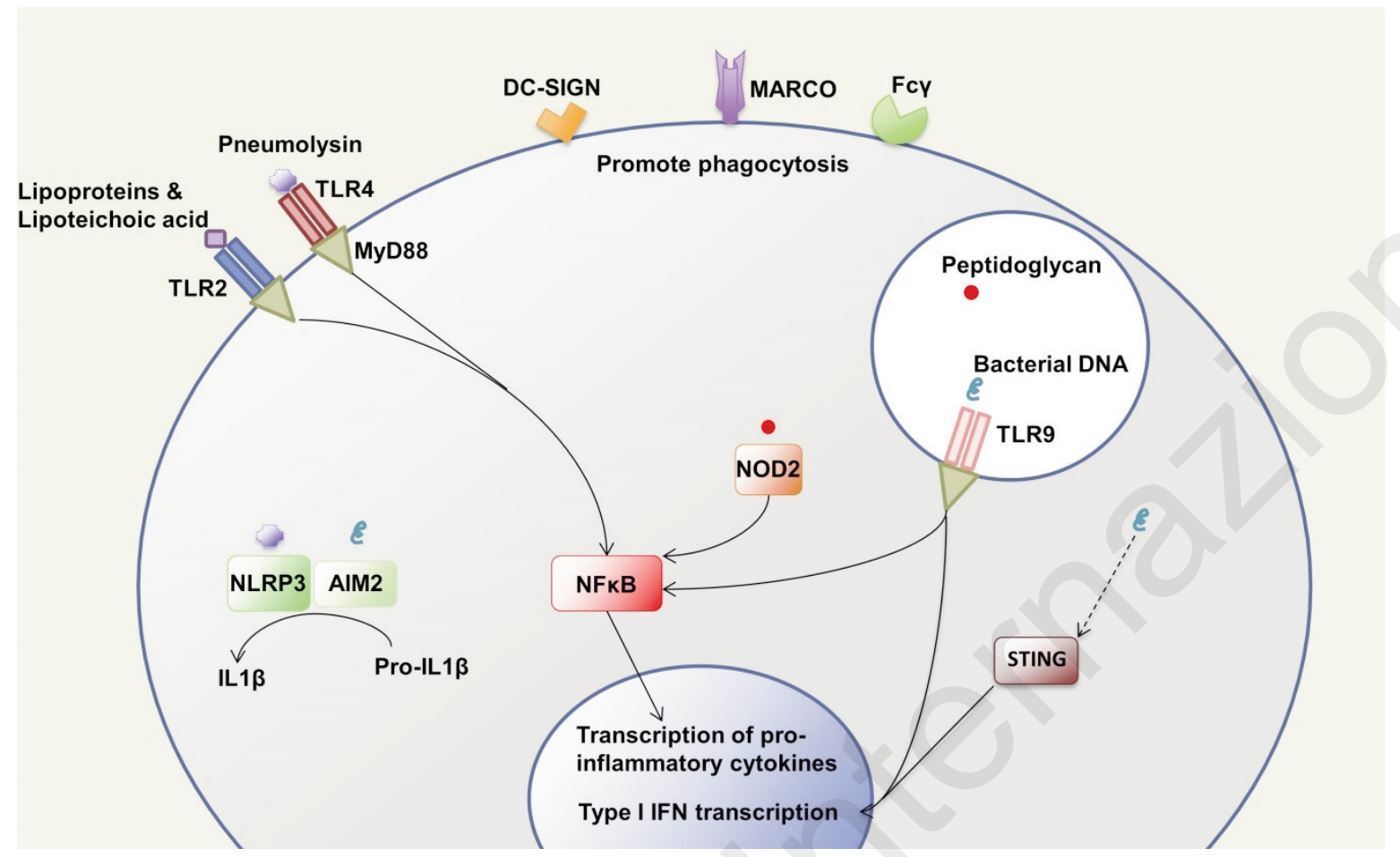

Figure 1 - PRR that interact with $S$. pneumoniae.

Several PRR (pattern recognition receptors) recognise S. pneumoniae. Non-opsonic phagocytic receptors like DC-SIGN (Dendritic Cell-Specific Intercellular adhesion molecule-3-Grabbing Non-integrin) and MARCO (Macrophage receptor with collagenous structure) recognise components of $S$. pneumoniae and induce its phagocytosis. Opsonic receptors like Fcy receptors recognise antibody that has bound $S$. pneumoniae, and again induce phagocytosis. TLRs (Toll like receptors) are cell surface and endosomal receptors that trigger intracellular signalling cascades to stimulate transcriptional events. TLR2 bind lipoproteins and TLR4 pneumolysin to induce NFKB translocation into the nucleus and the transcription of pro-inflammatory cytokines like TNF, IL1 $\beta$, and IL6. Cytoplasmic PRR like NOD2 (Nucleotide-binding oligomerization domain-containing protein 2) recognise peptidoglycan from $S$. pneumoniae cell wall and also activate NFKB. Bacterial DNA is recognised directly by TLR9 and indirectly by cytoplasmic signalling molecules like STING (Stimulator of interferon genes). Signalling cascades downstream of these events induce type I interferon transcription. The inflammasome components NLRP3 (NOD-like receptor family, pyrin domain containing 3) and AIM2 (Absent in melanoma 2) are activated by pneumolysin dependent mechanisms and bacterial DNA respectively to modify pro-cytokines into their secreted functional forms.

tory responses in humans (40).

TLR4 is usually stimulated by lipopolysaccharide from Gram negative bacterial cell walls, but may also be important for inflammatory response to $S$. pneumoniae perhaps through direct stimulation by the toxin pneumolysin $(40,44)$. TLR9 recognises the unmethylated CpG motifs of prokaryotic organisms and downstream signalling causes transcription of pro-inflammatory cytokines and type I interferons. The absence of TLR9 in mice results in reduced survival during $S$. pneumoniae infection, probably because TLR9 activation improves bacterial phagocytosis by alveolar macrophages (45).

There is built-in redundancy within the TLR system, with greater effects on morbidity and mortality when TLR signalling adaptor molecules are affected than after deletion of individual TLR abnormalities. For example absence of MyD88, an adaptor protein that is common to the function of all TLRs except for TLR3, is associated with worse outcomes of $S$. pneumoniae in- fection in mouse models of CNS infection, nasopharyngeal colonisation, pneumonia and sepsis than individual absence of TLR2 or TLR4 $(46,47)$. Additionally, IRAK4 deficiency results in a blunted pro-inflammatory cytokine secretion profile and increased mortality in both mice and humans during $S$. pneumoniae infection $(41,48)$, whereas TLR2 deficiency alone has minimal effects on mortality in mouse models of pneumonia (49).

\section{Cytosolic PRRs}

NOD like receptors (NLRs) are a group of intracellular, primarily cytosol-based PRRs (50). NOD2 is expressed on leucocytes and epithelial cells (51) and recognises muramyl dipeptide released from the $S$. pneumoniae cell wall during lysozyme-dependent digestion of bacteria in the phagolysosome (52), causing NFKB activation and subsequent pro-inflammatory 
gene transcription (53). Within macrophages, cytosolic protein complexes called inflammasomes also promote inflammation by activating the cytokines IL1 $\beta$ and IL18. NLRP3, a key protein of the inflammasome, is activated by pneumolysin and cell wall constituents released by $S$. pneumoniae (54-56), causing greater release of IL1 $\beta$. There is also evidence that cytoplasmic receptors can recognise $S$. pneumoniae DNA to elicit a type I IFN response. Type I IFN is usually associated with immunity to viral infection but seems to have a significant but poorly understood role in improving innate immune responses against $S$. pneumoniae, perhaps mediated by release of the chemokine RANTES and preventing bacterial translocation across epithelial and endothelial cells (57-59).

\section{Lectins and scavenger non-opsonic phagocytic receptors}

Lectins are carbohydrate binding proteins that recognise sugar moieties. Primarily they recognise mannose, fucose, and glucan structures (60). DC-SIGN (dendritic cell-specific ICAM-3 grabbing non integrin) is a human C-type lectin which binds mannose containing ligands and is expressed by macrophages and dendritic cells (61). In mouse models of $S$. pneumoniae infection SIGNR1, the mouse homologue of DCSIGN, binds to the $S$. pneumoniae capsule, promotes macrophage phagocytosis, and is protective in a mouse model of pneumonia $(62,63)$. Scavenger receptors are a range of structurally varied PRRs that recognise negatively charged macromolecules through shared similarities in shape and electrostatic charge distribution of their binding sites (64). Alveolar macrophages express the scavenger receptors MAR$\mathrm{CO}$ (Macrophage receptor with collagenous structure) and/or scavenger receptor A (SR-A). Both receptors have been implicated in pulmonary immunity against $S$. pneumoniae with reduced bacterial clearance in from the lungs in $\mathrm{MARCO}^{-/-}(65)$ and SR-A ${ }^{-/-}$mice (66). Macrophages isolated from $\mathrm{MARCO}^{-/-}$mice show reduced phagocytosis of $S$. pneumoniae, and MARCO activation also appears to amplify the inflammatory response to TLR2 or NOD2 stimulation (67). Further- more, MARCO appears to be downregulated on alveolar macrophages after influenza infection, contributing to secondary pneumococcal infection (61). Hence, MARCO seems to have a particularly important role for alveolar macrophage mediated immunity to $S$. pneumoniae lung infection.

\section{Acute phase response}

The local inflammatory response to $S$. pneumoniae infection in the lungs stimulates a systemic response characterised by increased production of multiple proteins from the liver, many of which play a role in host defence (68) (Table 2). This is termed the acute phase response, and is vital for effective host defence against $S$. pneumoniae. The acute phase response is largely mediated by the cytokine IL6, which is produced in large quantities during $S$. pneumoniae pneumonia and reaches the liver via the circulation (69). Exactly how the acute phase responses boosts immunity is not clear; the large increases in circulating levels of complement
The acute phase response is largely mediated by the cytokine IL6, which is produced in large quantity during $\mathrm{S}$. pneumoniae pneumonia and reaches the liver via the circulation. proteins, serum amyloid $P$ and

$C$ reactive protein associated with the acute phase response should improve recognition of $S$. pneumoniae and therefore neutrophil phagocytosis. Complement is a series of host proteins found in serum, epithelial lining fluid and cell surfaces that form protease cascades. When activated by the host proteins $C$ reactive protein, serum amyloid $\mathrm{P}$, or the complement component $\mathrm{C} 1 \mathrm{q}$ binding to the $S$. pneumoniae surface, these protease cascades result in the coating of the bacteria with $\mathrm{C} 3 \mathrm{~b}$ and $\mathrm{iC} 3 \mathrm{~b}$ molecules, thereby promoting bacterial phagocytosis via complement receptors $(6,35)$. The recognition of $S$. pneumoniae antigens by antibody is also a major activator of complement, and is described in more detail in the adaptive immune response section below. In addition induction of fever may inhibit optimal bacterial growth. Furthermore, increased per-

Table 2 - Components of the acute phase response that play a role in host defence.

\begin{tabular}{|c|c|}
\hline Component & Role in host defence \\
\hline Complement components: & Opsonisation for phagocytosis \\
\hline $\begin{array}{l}\text { C3,C4,C9, } \\
\text { Mannose binding lectin }\end{array}$ & Binding of antibody bound to pathogen to effect bacterial agglutination or lysis (35) \\
\hline $\mathrm{C}$ reactive protein & $\begin{array}{l}\text { Binds to phosphocholine - increasing agglutination, allowing complement binding } \\
\text { and phagocytosis ( } 70)\end{array}$ \\
\hline Serum amyloid $\mathrm{P}$ & $\begin{array}{l}\text { Binds to negatively charged carbohydrates - allowing complement binding and } \\
\text { phagocytosis }(71)\end{array}$ \\
\hline $\begin{array}{l}\text { Granulocyte Colony Stimulating } \\
\text { Factor }\end{array}$ & Increases granulopoesis and activates mature granulocytes \\
\hline $\begin{array}{l}\text { Coagulation components: } \\
\text { e.g. Fibrinogen }\end{array}$ & Involved in tissue repair and local containment of bacterial infection (72) \\
\hline$a_{1}$ protease inhibitor & Inhibit proteolytic enzymes and reduces inflammation-induced damage \\
\hline
\end{tabular}


meability of the endothelium allows some of these plasma constituents and cellular infiltrate into affected tissues, thereby probably promoting immune clearance within the lung during $S$. pneumoniae infection.

\section{Adaptive immune response}

The innate immune response to invading pathogens is generic, able to target conserved microbial struc-

tures without the host having

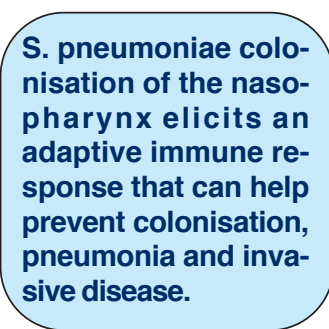

had prior exposure to that pathogen. In contrast, the adaptive immune system provides a highly specific immune response that is initiated only after exposure to the pathogen. The adaptive response is highly specific, depending on recognition of specific molecules expressed by pathogens (termed antigens), and provides the optimum protection against an invading pathogen. Generating an adaptive immune response can take days after initial exposure to pathogen, but also generates memory cells, which enable a more rapid and powerful response when that pathogen is subsequently re-encountered. S. pneumoniae colonisation of the nasopharynx elicits an adaptive immune response that can help prevent colonisation, pneumonia and invasive disease. Conversely, immune deficiencies that cause defects in the adaptive immune response such as antibody deficiency (73) and T cell deficiencies (74) lead to increased rates of $S$. pneumoniae pneumonia.

\section{B lymphocyte and antibody}

Prior infection will lead to induction of an antibody response by $\mathrm{B}$ lymphocytes to specific bacterial antigens. The high incidence of lung infections in subjects with antibody deficiencies and the efficacy of antibody-inducing vaccines at preventing $S$. pneumoniae pneumonia in children demonstrate a key role for antibody for protection against $S$. pneumoniae. In addition, the turning point described by $19^{\text {th }}$ and early $20^{\text {th }}$ century accounts of pneumonia when patients started to improve potentially reflect the point when the immune system has generated capsular serotype specific antibody. Antibody binding to $S$. pneumoniae improves immunity by activating complement deposition on the bacteria, so promoting complement-mediated phagocytosis, and by direct promotion of phagocytosis through recognition of the antibody by Fcy receptors expressed by macrophages and neutrophils. In addition, antibody can cause bacterial agglutination which improves removal of $S$. pneumoniae from the respiratory tract by the physical defences (75), and is likely to be how the present vaccine used in children can prevent nasopharyngeal.

$S$. pneumoniae antigens recognised by naturally ac- quired (via colonisation or previous infection) or vaccine-induced adaptive immunity include a range of bacterial proteins and the polysaccharide capsule. Polysaccharide antigens such as the $S$. pneumoniae capsule generate a weak adaptive immune response as these antigens are unable to recruit $T$ lymphocytes to assist B cell antibody responses. Although this type of $T$ cell-independent activation generates rapid $B$ cell proliferation and antibody production, it does not generate a memory $\mathrm{B}$ cell population that is required for a longer term and recall response on reinfection or revaccination (76). Furthermore, due to immaturity of the immune system, vaccination with capsular polysaccharide alone is ineffective in infants. In contrast, protein antigens stimulate a T-cell dependent B lymphocyte antibody response that generates a memory $B$ cell population and can therefore lead to a recall response to revaccination or infection, and are effective in infants. This effect of protein antigens has been exploited to overcome the limitations of polysaccharide as an immunogen; recent $S$. pneumoniae vaccines conjugate capsular polysaccharide to carrier protein adjuvants to induce a $\mathrm{T}$ cell dependent antibody response to the capsular antigen. As a consequence, the conjugated vaccine elicits protective antibody responses to $S$. pneumoniae capsular polysaccharide in infants and has been very effective at preventing $S$. pneumoniae infections, including pneumonia, caused by the serotypes covered by the vaccine (77).

Immunity to $S$. pneumoniae after nasopharyngeal colonisation in mice is dependent on antibody that recognises several $S$. pneumoniae cell wall proteins (78). Antibodies to these protein antigens are also present in human sera and may contribute towards naturally acquired immunity, but it is unclear at present whether antibody responses to the capsule or to a range of protein antigens are the dominant component of protective antibody responses in humans. Several of these protein antigens are at least partially conserved between all S. pneumoniae and could form the basis of a novel vaccine, especially if protein antigens are used in combination (79).

\section{T Iymphocytes}

Cellular adaptive immune responses are driven by $\mathrm{T}$ lymphocytes, which fall into 2 broad categories: CD4 expressing helper $\mathrm{T}$ cells that secrete cytokines to affect cells in the local milieu, and CD8 expressing cytotoxic $T$ cells that cause apoptosis of host cells infected with intracellular pathogens such as viruses. It is only recently that the importance of cellular responses for adaptive immunity against $S$. pneumoniae infection has been recognised (80).

\section{CD4 Th1 cellular immunity}

Th1 cells are a subset of helper $\mathrm{T}$ lymphocytes that secrete IFNY, the effect of which is to 'activate' macrophages to enhance intracellular killing. Their importance during $S$. pneumoniae lung infection is not clear, although the central role of alveolar macro-phages 


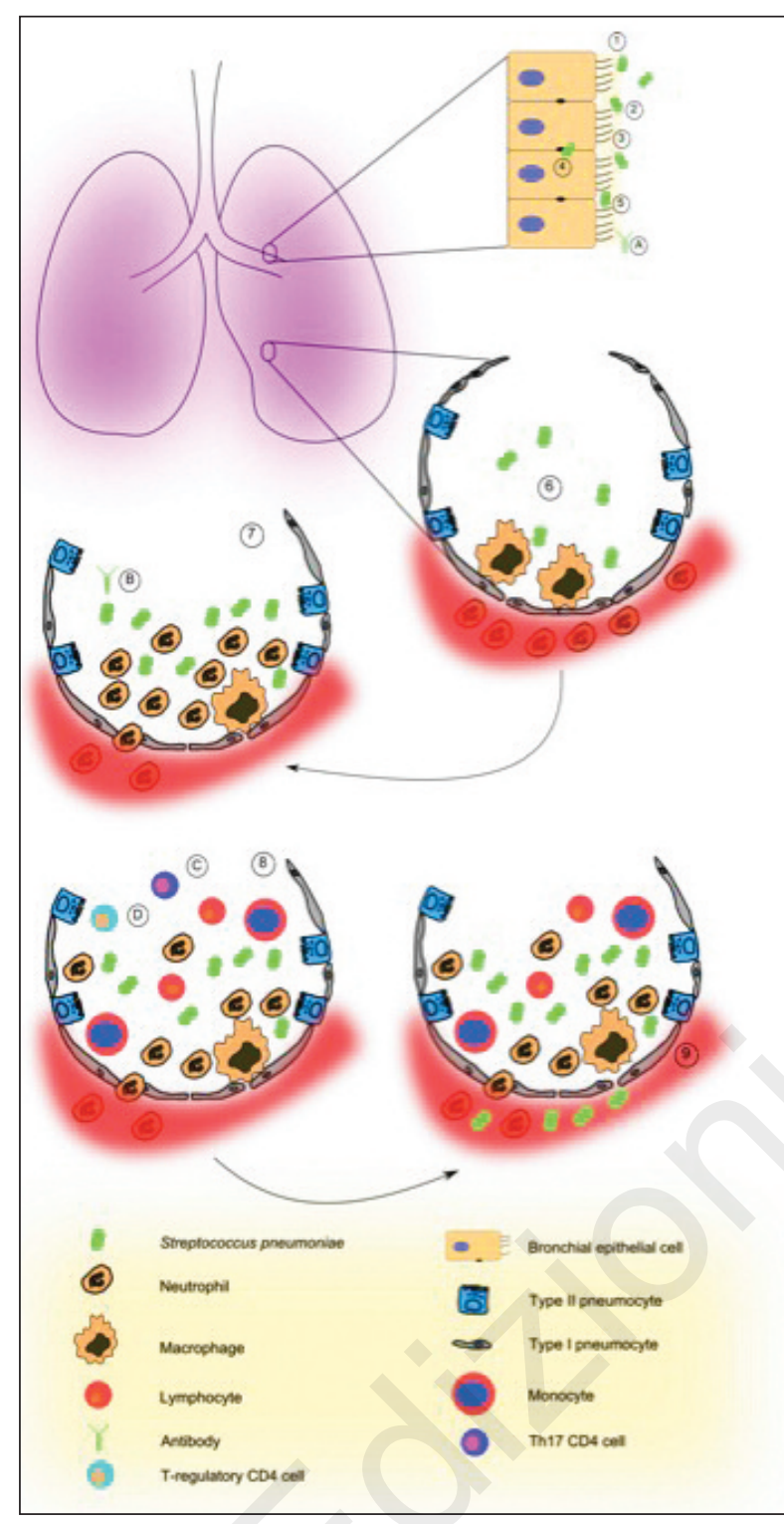

for control of infection would suggest Th1 adaptive responses should be beneficial. Human monocyte/T cell co-culture with live $S$. pneumoniae does lead to a Th1 cytokine response (81), and the deficiency of the cytokine IL12 (which stimulates Th1 cell proliferation) increases risk of $S$. pneumoniae lung infection (82).

\section{CD4 Th2 cellular immunity}

The Th2 subset of CD4 cells secrete IL4, 5 and 13 and improve $B$ cell responses to antigens associated with mucosal disease, however their role in adaptive immune response to $S$. pneumoniae has not been well characterised.

\section{CD4 Th17 cellular immunity}

CD4 Th17 cells indirectly improve immunity against mainly extracellular pathogens on mucosal surfaces. After recognition of an invading pathogen, Th17 cells secrete the cytokines IL17, IL17F, and IL22 to improved
Figure 2 - Schema of confirmed and likely mechanisms of lung immunity to invading $S$. pneumoniae.

Microaspiration of $S$. pneumoniae colonising the nasophar$y n x$ allows the bacteria to reach the lungs. There infection is most often controlled by the host's pulmonary innate immune mechanisms. These include the mucosal defences of: (1) Electrostatic repulsion between bacteria and mucous prevents bacteria reaching the mucosal surface; (2) and (3) clearance of bacteria by the mucociliary escalator with the motile cilia moving bacteria imbedded into the mucous layer to the pharynx; (4) tight junctions between the epithelial cells prevent transmigration of the bacteria; and (5) direct killing of the bacteria by lysozyme, lactoferrin, and cationic peptides present in the airway lining fluid. In the airspaces alveolar macrophages (6) are the first immune phagocytes to recognise and kill the pathogen, aided by complement and surfactant. Failure of these initial host defence mechanisms results in replication of the bacteria and the (7) co-ordinated secretion of cytokine and chemokines by epithelial cells and macrophages. This inflammatory response causes rapid recruitment to the alveoli of (8) neutrophils, which kill $S$. pneumoniae by phagocytosis, and (9) exudate containing complement and antibody to improve opsonisation and phagocytosis of the bacteria. Later, monocytes and lymphocytes are recruited to the lungs to assist with bacterial clearing and resolution of inflammation (8). Failure to control bacterial replication in the lung may result in invasive disease whereby the bacteria enter the blood causing septicaemia (9). Adaptive immune responses improve protection against $S$. pneumoniae at multiple levels, usually involving interactions with innate immune mechanisms. These include: (A) antibody mediated agglutination of $S$. pneumoniae improving the rate of bacterial removal by the mucociliary escalator; (B) antibody also improves alveolar macrophage and neutrophil phagocytosis directly through Fc gamma receptors or by increasing complement activity against $S$. pneumoniae; (C) Th17 CD4 cells improve rapid recruitment of neutrophils to the lungs during $S$. pneumoniae pneumonia, and possibly improve epithelial integrity and increase secretion of defensins; (D) T-regulatory CD4 cells help regulate inflammatory responses and thereby may help preserve epithelial barrier integrity and prevent septicaemia.

mucosal immunity. For example, IL17 induces bronchial epithelium to produce the chemokine CXCL8, resulting in neutrophil recruitment to the site of infection, and $\beta$ defensins increasing direct antimicrobial activity. In addition, Th17 responses improve epithelial barrier integrity and increase export of immunoglobulins to mucosal surfaces. Patients with hyper IgE syndrome have defects in Th17 cell differentiation and have increased susceptibility to $S$. pneumoniae pneumonia (83), and experimental human colonisation with $S$. pneumoniae results in detectable Th17 responses in bronchoalveolar fluid and blood (84). These data suggest an important role for CD4 Th17 cells for immunity to S. pneumoniae, and this has been confirmed in animal models in which CD4 Th17 responses to colonisation or vaccination can prevent $S$. pneumoniae nasopharyngeal colonisation and pneumonia $(85,86)$. CD4 Th17 cells recognise $S$. pneumoniae protein antigens rather than the CD4 Th17 cells recognise $S$. pneumoniae protein 
antigens rather than capsular polysaccharide, possibly inducing cross erotype protection (87).

\section{CD4 $T$ regulatory cells}

Tregulatory cells are largely anti-inflammatory, limiting excessive inflammation by secreting IL10 and reducing IFNy production. Treg cells are usually associated with poorer outcomes during infection, but recent data suggest they are actually beneficial during $S$. pneumoniae pneumonia. The proposed mechanism is that regulation of the inflammatory response during $S$. pneumoniae pneumonia helps prevent the bacteria from penetrating the epithelial layers to reach the blood and cause septicaemia (88).

\section{CD8 T cells}

As CD8 cells are usually associated with responses to intracellular organisms, they are generally thought not to have a role versus $S$. pneumoniae. However, genetic mutations in humans affecting TAP proteins required for CD8 function are associated with an increased incidence of $S$. pneumoniae infection (89), and in one mouse model of serotype $3 S$. pneumoniae lung infection survival was improved by CD8 positive cells. These data indicate there might be some role for CD8 cells for adaptive immunity to $S$. pneumoniae that requires further characterisation.

\section{Conclusion}

The immune mechanisms involved in preventing $S$. pneumoniae lung infections are complex (Figure 2) and incompletely explored. The mucociliary escalator and mucosal antibacterial proteins and peptides inhibit bacterial adherence and survival on the respiratory epithelium. These innate immune mechanisms are supported by alveolar macrophages that attempt to contain infection by phagocytosis whilst avoiding an unnecessarily strong inflammatory response. If this is unsuccessful, inflammation mediated by the alveolar macrophages and respiratory epithelium recruit neutrophils and monocytes to the lungs thereby increasing phagocytic capacity, and cause an acute phase response and alveolar exudate. Both macrophage and neutrophil phagocytosis is enhanced by the complement molecules and antibody recruited to the site of infection by the
A detailed understanding of the interactions between $\mathrm{S}$. pneumonia and host innate and adaptive responses is important for understanding the pathophysiology of S. pneumoniae pneumonia and the development of novel therapeutic and preventative strategies. inflammatory response. More recent data has shown this schema is relatively simplistic, and that are additional important (and sometimes seemingly contradictory) roles for adaptive cellular immunity, particularly Th17 and Treg cells. A detailed understanding of the interactions between $S$. pneumoniae and host innate and adaptive immune responses is important for understanding the pathophysiology of $S$. pneumoniae pneu- monia, and may lead to the development of novel therapeutic and preventative strategies.

\section{References}

1. O'Brien KL, et al. Burden of disease caused by Streptococcus pneumoniae in children younger than 5 years: global estimates. Lancet. 2009;374(9693): 893-902.

2. Walker CL, et al. Global burden of childhood pneumonia and diarrhoea. Lancet. 2013;381(9875):140516.

3. Welte T, Torres A, Nathwani D. Clinical and economic burden of community-acquired pneumonia among adults in Europe. Thorax. 2012;67(1): 71-9.

4. van Hoek AJ, et al. The effect of underlying clinical conditions on the risk of developing invasive pneumococcal disease in England. The Journal of infection. 2012;65(1):17-24.

5. Goldblatt D, et al. Antibody responses to nasopharyngeal carriage of Streptococcus pneumoniae in adults: a longitudinal household study. The Journal of infectious diseases. 2005;192(3):387-93.

6. Hyams C, et al. The Streptococcus pneumoniae capsule inhibits complement activity and neutrophil phagocytosis by multiple mechanisms. Infection and immunity. 2010;78(2):704-15.

7. Weinberger DM, et al. Association of serotype with risk of death due to pneumococcal pneumonia: a meta-analysis. Clinical infectious diseases: an official publication of the Infectious Diseases Society of America. 2010;51(6):692-9.

8. Harboe ZB, et al. Pneumococcal serotypes and mortality following invasive pneumococcal disease: a population-based cohort study. PLoS medicine. 2009;6(5):e1000081.

9. Bogaert D, De Groot R, Hermans PW. Streptococcus pneumoniae colonisation: the key to pneumococcal disease. The Lancet Infectious Diseases. 2004; 4(3):144-54.

10. Zhang JR, et al. The polymeric immunoglobulin receptor translocates pneumococci across human nasopharyngeal epithelial cells. Cell. 2000;102(6):82737.

11. Cundell DR, et al. Streptococcus pneumoniae anchor to activated human cells by the receptor for plateletactivating factor. Nature. 1995;377(6548):435-8.

12. Clarke TB, et al. Invasive bacterial pathogens exploit TLR-mediated downregulation of tight junction components to facilitate translocation across the epithelium. Cell host \& microbe. 2011;9(5):404-14.

13. Kash JC, et al. Lethal synergism of 2009 pandemic H1N1 influenza virus and Streptococcus pneumoniae coinfection is associated with loss of murine lung repair responses. mBio. 2011; 2(5).

14. Nuorti JP, et al. Cigarette smoking and invasive pneumococcal disease. Active Bacterial Core Surveillance Team. The New England journal of medicine. 2000;342(10):681-9.

15. Shaper M, et al. PspA protects Streptococcus pneumoniae from killing by apolactoferrin, and antibody to 
PspA enhances killing of pneumococci by apolactoferrin [corrected]. Infection and immunity. 2004;72 (9):5031-40.

16. Davis KM, et al. Resistance to mucosal lysozyme compensates for the fitness deficit of peptidoglycan modifications by Streptococcus pneumoniae. PLoS pathogens. 2008;4(12): e1000241.

17. Lee HY, et al. Antimicrobial activity of innate immune molecules against Streptococcus pneumoniae, Moraxella catarrhalis and nontypeable Haemophilus influenzae. BMC infectious diseases. 2004;4:12.

18. Sorrentino $\mathrm{R}$, et al. Pattern recognition receptors and interleukin-8 mediate effects of Gram-positive and Gram-negative bacteria on lung epithelial cell function. British journal of pharmacology. 2008;154 (4):864-71.

19. Marriott HM, et al. Interleukin-1 beta regulates CXCL8 release and influences disease outcome in response to Streptococcus pneumoniae, defining intercellular cooperation between pulmonary epithelial cells and macrophages. Infection and immunity. 2012;80(3): 1140-9.

20. Sano H, et al. Regulation of inflammation and bacterial clearance by lung collectins. Respirology. 2006;11 Suppl:S46-50.

21. Wong A, et al. Welders are at increased risk for invasive pneumococcal disease. International journal of infectious diseases: IJID: official publication of the International Society for Infectious Diseases. 2010;14 (9):e796-9.

22. Phipps JC, et al. Cigarette smoke exposure impairs pulmonary bacterial clearance and alveolar macrophage complement-mediated phagocytosis of Streptococcus pneumoniae. Infection and immunity. 2010;78(3):1214-20.

23. Antonini JM, et al. Effect of short-term stainless steel welding fume inhalation exposure on lung inflammation, injury, and defense responses in rats. Toxicology and applied pharmacology. 2007;223(3):234-45.

24. Ghoneim HE, Thomas PG, McCullers JA. Depletion of alveolar macrophages during influenza infection facilitates bacterial superinfections. Journal of immunology. 2013;191(3):1250-9.

25. Mogensen TH, et al. Mechanisms of dexamethasone-mediated inhibition of Toll-like receptor signaling induced by Neisseria meningitidis and Streptococcus pneumoniae. Infection and immunity. 2008;76(1):189-97.

26. Gwyer Findlay E, et al. OX40L blockade is therapeutic in arthritis, despite promoting osteoclastogenesis. Proceedings of the National Academy of Sciences of the United States of America. 2014;111(6):2289-94.

27. Dockrell DH, et al. Alveolar macrophage apoptosis contributes to pneumococcal clearance in a resolving model of pulmonary infection. Journal of immunology. 2003;171(10):5380-8.

28. Fang $R$, et al. Critical roles of ASC inflammasomes in caspase-1 activation and host innate resistance to Streptococcus pneumoniae infection. Journal of immunology. 2011;187(9):4890-9.

29. Knapp S, et al. Alveolar macrophages have a protective antiinflammatory role during murine pneumo- coccal pneumonia. American journal of respiratory and critical care medicine. 2003;167(2):171-9.

30. Marriott HM, et al. Decreased alveolar macrophage apoptosis is associated with increased pulmonary inflammation in a murine model of pneumococcal pneumonia. Journal of immunology. 2006;177(9): 6480-8.

31. Cox G, Crossley J, Xing Z. Macrophage engulfment of apoptotic neutrophils contributes to the resolution of acute pulmonary inflammation in vivo. American journal of respiratory cell and molecular biology. 1995;12(2):232-7.

32. Kumashi P, et al. Streptococcus pneumoniae bacteremia in patients with cancer: disease characteristics and outcomes in the era of escalating drug resistance (1998-2002). Medicine (Baltimore). 2005;84(5): 303-12.

33. Carratala J, et al. Bacteremic pneumonia in neutropenic patients with cancer: causes, empirical antibiotic therapy, and outcome. Archives of internal medicine. 1998;158(8):868-72.

34. Marks $M$, et al. Influence of neutropenia on the course of serotype 8 pneumococcal pneumonia in mice. Infection and immunity. 2007;75(4):1586-97.

35. Brown JS, et al. The classical pathway is the dominant complement pathway required for innate immunity to Streptococcus pneumoniae infection in mice. Proceedings of the National Academy of Sciences of the United States of America. 2002;99(26):16969-74.

36. Beiter K, et al. An endonuclease allows Streptococcus pneumoniae to escape from neutrophil extracellular traps. Current biology: CB. 2006;16(4):401-7.

37. Standish AJ, Weiser JN. Human neutrophils kill Streptococcus pneumoniae via serine proteases. Journal of immunology. 2009;183(4):2602-9.

38. Hahn I, et al. Cathepsin $G$ and neutrophil elastase play critical and nonredundant roles in lung-protective immunity against Streptococcus pneumoniae in mice. Infection and immunity. 2011;79(12):4893-901.

39. Srivastava A, et al. Recombinant bactericidal/permeability-increasing protein rBPI21 protects against pneumococcal disease. Infection and immunity. 2007;75(1):342-9.

40. Tomlinson G, et al. TLR-Mediated Inflammatory Responses to Streptococcus pneumoniae Are Highly Dependent on Surface Expression of Bacterial Lipoproteins. Journal of immunology. 2014.

41. $\mathrm{Ku} \mathrm{CL}$, et al. IRAK4 and NEMO mutations in otherwise healthy children with recurrent invasive pneumococcal disease. Journal of medical genetics. 2007;44(1):16-23.

42. Schenk M, Belisle JT, Modlin RL. TLR2 looks at lipoproteins. Immunity. 2009;31(6):847-9.

43. Chimalapati S, et al. Effects of deletion of the Streptococcus pneumoniae lipoprotein diacylglyceryl transferase gene lgt on $\mathrm{ABC}$ transporter function and on growth in vivo. PloS one. 2012;7(7):e41393.

44. Malley R, et al. Recognition of pneumolysin by Tolllike receptor 4 confers resistance to pneumococcal infection. Proceedings of the National Academy of Sciences of the United States of America. 2003;100 (4):1966-71. 
45. Albiger B, et al. Toll-like receptor 9 acts at an early stage in host defence against pneumococcal infection. Cellular Microbiology. 2007;9(3):633-44.

46. Albiger B, et al. Myeloid differentiation factor 88-dependent signalling controls bacterial growth during colonization and systemic pneumococcal disease in mice. Cellular Microbiology. 2005;7(11):1603-15.

47. Koedel $U$, et al. MyD88 is required for mounting a robust host immune response to Streptococcus pneumoniae in the CNS. Brain: a journal of neurology. 2004;127(Pt 6):1437-45.

48. Pennini ME, et al. Complete dependence on IRAK4 kinase activity in TLR2, but not TLR4, signaling pathways underlies decreased cytokine production and increased susceptibility to Streptococcus pneumoniae infection in IRAK4 kinase-inactive mice. Journal of immunology. 2013;190(1):307-16.

49. Knapp S, et al. Toll-like receptor 2 plays a role in the early inflammatory response to murine pneumococcal pneumonia but does not contribute to antibacterial defense. Journal of immunology. 2004;172(5): 3132-8.

50. Koppe U, Suttorp N, Opitz B. Recognition of Streptococcus pneumoniae by the innate immune system. Cellular Microbiology. 2012;14(4):460-6.

51. Opitz B, et al. Nucleotide-binding oligomerization domain proteins are innate immune receptors for internalized Streptococcus pneumoniae. The Journal of biological chemistry. 2004;279(35):36426-32.

52. Davis KM, Nakamura S, Weiser JN. Nod2 sensing of lysozyme-digested peptidoglycan promotes macrophage recruitment and clearance of S. pneumoniae colonization in mice. The Journal of clinical investigation. 2011;121(9):3666-76.

53. Parker D, Prince A. Innate immunity in the respiratory epithelium. American journal of respiratory cell and molecular biology. 2011;45(2):189-201.

54. Witzenrath M, et al. The NLRP3 inflammasome is differentially activated by pneumolysin variants and contributes to host defense in pneumococcal pneumonia. Journal of immunology. 2011;187(1):434-40.

55. McNeela EA, et al. Pneumolysin activates the NLRP3 inflammasome and promotes proinflammatory cytokines independently of TLR4. PLoS pathogens. 2010;6(11):e1001191.

56. Martinon F, et al. Identification of bacterial muramyl dipeptide as activator of the NALP3/cryopyrin inflammasome. Current biology: CB. 2004;14(21): 1929-34.

57. LeMessurier KS, et al. Type I interferon protects against pneumococcal invasive disease by inhibiting bacterial transmigration across the lung. PLoS pathogens. 2013;9(11):e1003727.

58. Koppe U, et al. Streptococcus pneumoniae stimulates a STING- and IFN regulatory factor 3-dependent type I IFN production in macrophages, which regulates RANTES production in macrophages, cocultured alveolar epithelial cells, and mouse lungs. Journal of immunology. 2012;188(2):811-7.

59. Parker D, et al. Streptococcus pneumoniae DNA initiates type I interferon signaling in the respiratory tract. mBio. 2011;2(3):e00016-11.
60. Geijtenbeek TB, Gringhuis SI. Signalling through Ctype lectin receptors: shaping immune responses. Nature reviews. Immunology. 2009;9(7):465-79.

61. Pluddemann A, Mukhopadhyay S, Gordon S. Innate immunity to intracellular pathogens: macrophage receptors and responses to microbial entry. Immunological reviews. 2011;240(1):11-24.

62. Lanoue A, et al. SIGN-R1 contributes to protection against lethal pneumococcal infection in mice. The Journal of experimental medicine. 2004;200(11): 1383-93.

63. Koppel EA, et al. Interaction of SIGNR1 expressed by marginal zone macrophages with marginal zone $B$ cells is essential to early lgM responses against Streptococcus pneumoniae. Molecular immunology. 2008;45(10):2881-7.

64. Canton J, Neculai D, Grinstein S. Scavenger receptors in homeostasis and immunity. Nature reviews. Immunology. 2013;13(9):621-34.

65. Arredouani $\mathrm{M}$, et al. The scavenger receptor MARCO is required for lung defense against pneumococcal pneumonia and inhaled particles. The Journal of experimental medicine. 2004;200(2):267-72.

66. Arredouani MS, et al. The macrophage scavenger receptor SR-AI/II and lung defense against pneumococci and particles. American journal of respiratory cell and molecular biology. 2006;35(4):474-8.

67. Dorrington MG, et al. MARCO is required for TLR2and Nod2-mediated responses to Streptococcus pneumoniae and clearance of pneumococcal colonization in the murine nasopharynx. Journal of immunology. 2013;190(1):250-8.

68. Gabay C, Kushner I. Acute-phase proteins and other systemic responses to inflammation. The New England journal of medicine. 1999;340(6):448-54.

69. Quinton LJ, et al. Mechanisms of the hepatic acutephase response during bacterial pneumonia. Infection and immunity. 2009;77(6):2417-26.

70. Simons JP, et al. C-reactive protein is essential for innate resistance to pneumococcal infection. Immunology. 2014;142(3):414-20.

71. Yuste J, et al. Additive inhibition of complement deposition by pneumolysin and PspA facilitates Streptococcus pneumoniae septicemia. Journal of immunology. 2005;175(3):1813-9.

72. Yang $\mathrm{H}$, et al. Interleukin-1 promotes coagulation, which is necessary for protective immunity in the lung against Streptococcus pneumoniae infection. The Journal of infectious diseases. 2013;207(1):50-60.

73. Martinot $\mathrm{M}$, et al. Immunoglobulin deficiency in patients with Streptococcus pneumoniae or Haemophilus influenzae invasive infections. International journal of infectious diseases : IJID: official publication of the International Society for Infectious Diseases. 2014;19:79-84.

74. Klein C, et al. Major histocompatibility complex class Il deficiency: clinical manifestations, immunologic features, and outcome. The Journal of pediatrics. 1993;123(6):921-8.

75. Roche AM, et al. Antibody blocks acquisition of bacterial colonization through agglutination. Mucosal Immunol. 2014. 
76. Coutinho A, Moller G. B cell mitogenic properties of thymus-independent antigens. Nature: New biology. 1973;245(140):12-4.

77. Simonsen L, et al. Effect of 13-valent pneumococcal conjugate vaccine on admissions to hospital 2 years after its introduction in the USA: a time series analysis. Lancet Respir Med. 2014;2(5):387-94.

78. Cohen JM, et al. Protective contributions against invasive Streptococcus pneumoniae pneumonia of antibody and Th17-cell responses to nasopharyngeal colonisation. PloS one. 2011;6(10):e25558.

79. Ogunniyi $A D$, et al. Immunization of mice with combinations of pneumococcal virulence proteins elicits enhanced protection against challenge with Streptococcus pneumoniae. Infection and immunity. 2000;68(5):3028-33.

80. Malley R, et al. CD4+ T cells mediate antibody-independent acquired immunity to pneumococcal colonization. Proceedings of the National Academy of Sciences of the United States of America. 2005;102 (13):4848-53.

81. Olliver M, et al. Human monocytes promote Th1 and Th17 responses to Streptococcus pneumoniae. Infection and immunity. 2011;79(10):4210-7.

82. Haraguchi $\mathrm{S}$, et al. Interleukin 12 deficiency associated with recurrent infections. Proceedings of the $\mathrm{Na}$ - tional Academy of Sciences of the United States of America. 1998;95(22):13125-9.

83. Milner JD, et al. Impaired $T(H) 17$ cell differentiation in subjects with autosomal dominant hyper-IgE syndrome. Nature. 2008;452(7188):773-6.

84. Wright AK, et al. Experimental human pneumococcal carriage augments IL-17A-dependent T-cell defence of the lung. PLoS pathogens. 2013;9(3):e1003274.

85. Zhang Z, Clarke TB, Weiser JN. Cellular effectors mediating Th17-dependent clearance of pneumococcal colonization in mice. The Journal of clinical investigation. 2009;119(7):1899-909.

86. Moffitt KL, et al. $T(H) 17$-based vaccine design for prevention of Streptococcus pneumoniae colonization. Cell host \& microbe. 2011;9(2):158-65.

87. Moffitt KL, Gierahn TM, Lu YJ, Gouveia P, Alderson M, Flechtner JB, Higgins DE, Malley R. T(H)17based vaccine design for prevention of Streptococcus pneumoniae colonization. Cell Host Microbe. 2011 Feb 17;9(2):158-65.

88. Neill DR, et al. T regulatory cells control susceptibility to invasive pneumococcal pneumonia in mice. PLoS pathogens. 2012;8(4):e1002660.

89. Gadola SD, et al. TAP deficiency syndrome. Clinical and experimental immunology. 2000;121(2): 173-8. 